\title{
Long-term Suboxone Treatment and its Benefit on Long-Term Remission for Opiate Dependence
}

Anish S Shah ${ }^{1}$, James Young ${ }^{2}$ and Karen Vieira ${ }^{3}$

11260 N Dutton Ave, Suite 275, Santa Rosa, CA, 95401, USA

2 Napa State Hospital, 2100 Napa Valleho Highway, Napa, CA, 94558, USA

${ }^{3}$ The Med Writers, 9314 Forest Hill Blvd Suite 6, West Palm Beach, FL, 33411, USA

*Corresponding author: Anish S Shah, MD, 1260 N Dutton Ave, Suite 275, Santa Rosa, Ca, USA, Tel: 707 696 8045 ; E-mail: ashah@siyanclinical.com Received Date: August 30, 2014, Accepted Date: October 8, 2014, Published Date: October 15, 2014

Copyright: ( 2014, Anish S Shah et al., This is an open-access article distributed under the terms of the Creative Commons Attribution License, which permits unrestricted use, distribution, and reproduction in any medium, provided the original author and source are credited.

\begin{abstract}
Aims: To review the current professional literature and describe factors related to Suboxone treatment that contributes to sustained opioid detoxification, as well as the characteristics of patients who are likely to benefit from long-term Suboxone treatment.

Methods: Clinical trials and Suboxone therapy research that evaluated the efficacy of Suboxone treatment in comparison to methadone, naltrexone, and clonidine in different settings (e.g., hospitals, community centers, and outpatient treatment) were thoroughly reviewed.

Results: Clinical studies have repeatedly demonstrated the superiority of Suboxone therapy as a treatment for opioid dependence in comparison to methadone, naltrexone, and clonidine. Research further indicates that longterm Suboxone treatment in an office-based setting for a minimum of four weeks yields the largest percentages of opioid-free urine samples, opioid detoxification, and treatment retention rates.

Conclusions: Factors such as the intensity of withdrawal symptoms, the presence of anxiety problems, no prior history of substance abuse, and being a non-smoker were found to be associated with successful opioid detoxification and sustained abstinence. Socio-demographic factors such as gender, race, age, level of education, or employment status were not found to be significant predictors of treatment success.
\end{abstract}

Keywords: Suboxone treatment; Drug replacement therapy; Opioid dependence; Opiate dependence; Opioid detoxification; Opiates

\section{Introduction}

Opiates are typically prescribed for pain relief and, during recent years, prescription opiate dependence has become one of the most prevalent forms of drug abuse $[1,2]$. Opiate dependence is often the result of the regular use of prescribed forms of opiates (e.g. oxycodone, hydrocodone, fentanyl, codeine, or morphine) or illegal drugs (e.g. heroin or opium) for extended periods of time. Certain groups of individuals are particularly susceptible to prescription opioid abuse and dependence. The groups include: chronic pain patients, especially those whose pain causes functional limitations; patients with a history of substance abuse (alcohol or illicit drugs); patients who have mental illnesses (e.g. mood disorder); and patients with a history of childhood sexual abuse or neglect [3-6].

Furthermore, misused opioid medications such as oxycodone and hydrocodone can produce effects similar to heroin. Research indicates that the abuse of these types of prescription medications may lead to abuse of the less expensive illegal drug, heroin, especially when prescription opiates are no longer available [7]. Additionally, according to the National Institute on Drug Abuse, in 2011, 4.2 million Americans over the age of 11 reported that they had used heroin at least once in their lives while approximately $23 \%$ of heroin users become dependent it [8]. Understanding the action of opioids and how to interfere with their function is critical to successful treatment regimens.

Opioids function by binding to mu receptors in the brain, leading to decreased pain perception and increased pain tolerance. However, chronic consumption results in diminishing returns, wherein the heightened level of pain tolerance decreases. This often leads a patient to consume higher or more frequent doses. This significant decline in opioid-induced activity is a sign of opioid dependence, and, when an individual becomes dependent on opiates, abrupt discontinuation can lead to intense withdrawal symptoms. Compounding the issue of opiate dependence, habitual opiate consumption leads to opioid intoxication, a sense of euphoria and additional pleasurable feelings. Opioid intoxication results from the binding of opioids to the mu receptors and subsequent release of the neurotransmitters that influence mood to $[9,10]$.

As a result of these complicating conditions, treatment for opioid dependence often involves a combination of psychological treatment or counseling and drug replacement therapy, which results in opioid detoxification. Detoxification is the professionally controlled and supervised withdrawal from a drug. One of the most innovative treatments from the last decade that shows promise is a prescription medication called Suboxone. Herein, we describe a systematic review of the effects of long-term Suboxone treatment in comparison to other forms of drug replacement therapy such as methadone, clonidine, or 
Page 2 of 4

naltrexone as well as the characteristics of patients that may benefit from long-term Suboxone treatment.

\section{Suboxone as a Drug Replacement Therapy}

Suboxone contains the substances buprenorphine and naloxone. Research regarding the underlying mechanism of buprenorphine is somewhat controversial in terms of whether it functions as a full opioid agonist or a partial opioid agonist, but several studies including reports as recent as 2014 indicate that buprenorphine is a partial opioid agonist [11-13]. Buprenorphine has a high affinity for mu receptors, which prevents full opioid agonists such as oxycodone or heroin from binding to the receptors. This form of replacement therapy has been shown to reduce withdrawal symptoms, cravings, and feelings of euphoria in some patients [13]. The second component of Suboxone, naloxone, is an opioid antagonist that functions as an abuse deterrent. The inclusion of naloxone in Suboxone decreases the likelihood of injection since naloxone negates any drug high that could be achieved by buprenorphine alone, thus decreasing the risk cases of injected buprenorphine-related deaths [14].

Several recent studies have shown that suboxone is a medically and cost effective treatment for heroin addiction [15-17]. Suboxone has seen consistent market growth over the years. For instance, in 2002 approximately 12,000 prescriptions were issued and this number rose to 300,000 in 2006 [18]. These statistics indicate that Suboxone treatment rates are consistently increasing due to its efficacy.

Clinical research has demonstrated the efficacy and safety of combining buprenorphine and naloxone for the treatment of opioid abuse. One study in particular involved 326 patients who received either a tablet containing buprenorphine $(16 \mathrm{mg})$ and naloxone (4 $\mathrm{mg}$ ), buprenorphine alone (16 mg), or a placebo daily for four weeks in an office-based setting. The patients reported to an office daily (Monday through Friday) and received approximately one hour of counseling each week. The results showed that larger percentages of patients who received the combination tablet or buprenorphine alone had more opioid-negative urine samples and less opioid cravings than the placebo group [19]. The efficacy of this method was attributed to treating the patients in an office-based setting in combination with individual counseling.

\section{Suboxone Treatment versus Other Treatment Modalities}

Medications such as methadone, naltrexone, and clonidine were commonly administered as treatments for opioid dependence prior to the use of Suboxone. Safety research that was conducted for naltrexone, methadone, and buprenorphine showed that patients who took naltrexone alone often experienced a relapse or opioid overdose within two weeks of discontinuing naltrexone [20].

Buprenorphine treatment has demonstrated a therapeutic efficacy that is comparable to methadone, but this approach entails induction, stabilization, and maintenance phases. Although comparable in efficacy, buprenorphine carries a lower risk of overdose [21]. Furthermore, it is important that buprenorphine therapy is initiated at the onset of withdrawal symptoms as research indicates that inpatients without a history of substance abuse that have high baseline withdrawal scores appear to benefit the most from long-term Suboxone treatment [22,23]. An additional disadvantage of buprenorphine therapy involves its potential inefficacy in individuals who required high methadone doses prior to buprenorphine treatment.

Methadone has been administered in the United States since 1947 to treat opioid dependence, but individuals who take this medication tend to have significantly high relapse rates. Methadone is a full opioid agonist and often produces effects that are very similar to the opiate that is it meant to counteract [11]. In addition to methadone, another medication called clonidine became a common form of opioid dependence treatment. Subsequent research indicated that detoxification by clonidine only resulted in a slight decrease in the rate of relapse [24], making a more effective form of drug replacement therapy necessary.

In the late 1990s clinical trials involving Subutex (buprenorphine) and Suboxone (buprenorphine and naloxone) began and the initial results indicated that these treatments were both safe and effective with diminishing side effects requiring ancillary medications [25]. In 2002, the Food and Drug Administration (FDA) approved the use of buprenorphine for opioid dependence treatment and this initiative led to substantive research.

In particular, The Clinical Trials Network conducted two studies that compared a 13-day opioid detoxification with Suboxone to clonidine: one with inpatients and one with outpatients. The objectives were to determine the number of patients with opioid-negative urine samples on days 13 and 14 as well as to assess the abstinence rates during the detoxification period and follow-up (1,3, and 6 months post-treatment) [24,25]. Treatment retention, patient satisfaction, withdrawal symptoms, and safety were also evaluated and counseling was provided during the detoxification period. The dose for each patient was gradually increased, with the maximum dose being $16 \mathrm{mg}$ of buprenorphine and $4 \mathrm{mg}$ of naloxone until tapering began. The results of the inpatient study demonstrated treatment success for $77 \%$ of the inpatients treated with Suboxone in comparison to $22 \%$ of the inpatients treated with clonidine. The treatment success for outpatients who were administered Suboxone was $29 \%$ in comparison to $5 \%$ of the outpatients who were administered clonidine [24]. Overall, inpatient treatment was slightly more effective than outpatient treatment. Additional large-scale, clinical trials evaluated predictors and mediators of treatment success for inpatients who received either Suboxone or clonidine. Contrary to what was hypothesized, higher rates of treatment success were observed for patients who had high baseline withdrawal scores [23]. More specifically, inpatients with high baseline withdrawal symptoms were four times more likely to achieve detoxification and sustained abstinence than inpatients with lower baseline withdrawal symptoms. There was also a significant effect in regards to medication type as the Suboxone group displayed greater reductions in withdrawal symptoms than the clonidine group, especially during the first days of treatment. Medication type was therefore, the strongest predictor of treatment efficacy. Non-daily heroin users also demonstrated larger percentages of sustained detoxification and abstinence in comparison to daily heroin users. Additional factors that were associated with treatment success included being a non-smoker and experiencing anxiety problems. Socio-demographic factors such as gender, race, age, level of education, or employment status were not found to be predictors of treatment success.

These types of clinical trials were initially conducted with adults and were repeated with adolescents after the effectiveness of Suboxone was substantiated. The efficacy of Suboxone treatment is mostly attributed to its ability to significantly reduce withdrawal symptoms 
and feelings of euphoria. Furthermore, the rate of abstinence significantly increases for both inpatients and outpatients who receive behavioral therapy in combination with Suboxone treatment $[26,27]$.

\section{The Benefits of Long-term Suboxone Treatment}

Studies have demonstrated that the length of time Suboxone is consumed further contributes to its effectiveness. For example, a comparison between a 5-day buprenorphine treatment period (one daily dose of $2 \mathrm{mg}$ ) and a 30-day Suboxone treatment period (daily blocking dose of $16 \mathrm{mg}$ buprenorphine; $4 \mathrm{mg}$ naloxone) showed that treatment success was greater for patients who were treated for 30 days [28]. Patients in the 30 -day treatment group were also more likely to enroll in longer-term treatment in order to maintain abstinence.

Physicians typically recommend cognitive behavioral therapy in combination with Suboxone treatment in order to address psychological issues that may have contributed to the dependence and to prevent relapse. Successful detoxification and behavioral therapy are highly associated with sustained opioid abstinence. Accordingly, participants who were in the 30-day treatment group attended more therapy sessions and submitted less opioid-positive urine samples than those in the 5-day group [28]. Research indicates, however, that once stabilization has occurred at approximately four weeks, the schedule that is chosen to taper patients off of Suboxone does not generally influence relapse rates.

A clinical trial that made a comparison of a 7-day and 28-day tapering regimen after a 4 -week stabilization period showed no significant difference in relapse rates or opioid-free urine samples between the groups [29]. For the both groups, Suboxone was gradually reduced and then discontinued at either day seven or day 28 . These results indicate that once stabilization has been successfully achieved, there is no advantage to prolonging the amount of time an individual is tapered off of Suboxone.

Studies currently show that the most common tapering schedule, after successful opioid detoxification has been achieved, is 14 days and that individuals who enroll in long-term Suboxone treatment are better able to maintain opioid-free stabilization after a one-year follow-up $[11,30]$. The stabilization phase begins when a patient is no longer suffering from withdrawal symptoms and is experiencing minimal to no side effects or opioid cravings. Therefore, clinical studies indicate that behavioral therapy and Suboxone treatment which lasts for a minimum of 30 days followed by a 14-day tapering schedule after stabilization is the most clinically effective for adults with opioid dependence. A study involving adolescents whose ages ranged from 15 to 21 indicated that administering Suboxone over a 12 week period, in which the medication is gradually reduced and then discontinued at week 12, produced better treatment outcomes than a 14-day tapering period [30]. However, relapse rates were high at follow-up visits $(6,9$, and 12 months) for the 14-day group as well as the 12 week group. Although relapse rates increased over time for both groups, adolescents who were in the 14-day detoxification group relapsed sooner and with greater severity than the 12 -week group. The 12-week Suboxone group also had lower percentages of opioidpositive urine samples at follow-up visits than the detoxification group and this was attributed to Suboxone patients who enrolled in longerterm treatments [15].

Despite the supporting evidence which shows that Suboxone treatment which is a minimum of 30 days for adults and 12 weeks for adolescents increases the rate of successful opioid detoxification, follow-up research indicates that abstinence rates tend to decrease as time progresses $[28,30]$. Therefore, maintenance regimens such as longer-term Suboxone treatments are necessary in order for recovering opioid dependent patients to remain abstinent. By the completion of most Suboxone detoxification periods, patients may be tapered down to $2 \mathrm{mg}$ of buprenorphine and $0.05 \mathrm{mg}$ of naloxone [24]. Maintaining these doses long-term may decrease the occurrence of relapse for recovering patients.

\section{Discussion}

Irrespective of whether an individual is dependent on prescription of illicit opiates, opioid dependency harms the society and the economy as much as it hurts the person who is suffering from the dependence. Loss of employment and heightened crime rates due to this type of drug abuse results in financial problems for both the government and taxpayers. Fortunately, the results of an opioid drug abuse survey showed that the employment rates for individuals who enroll in opioid substitution treatment programs dramatically improve as they progress through the program [31]. These individuals also tend to improve their work performance because their perception of tasks that were previously viewed as excessively challenging begins to change during treatment. Work-related issues such as mentally demanding assignments, unfavorable work schedules, and an intense work pace are also viewed as less of a burden as a result of opioid substitution treatment.

Overall, clinical studies have repeatedly demonstrated the efficacy of Suboxone therapy as a treatment for opioid dependence in comparison to other treatment modalities such as methadone, naltrexone, and clonidine $[11,23,24]$. Research further indicates that long-term Suboxone treatment in an office-based setting that is a minimum of four weeks yields the largest percentages of opioid-free urine samples, opioid detoxification, and treatment retention rates $[28,30]$. Being able to monitor the administration and dosage of Suboxone through inpatient treatment appears to further improve treatment retention rates as does attending behavioral therapy or counseling.

The observation that abstinence rates begin to gradually decrease at follow-up visits for patients who do not enroll in longer-term treatment programs demonstrates the need for a maintenance regimen in order to achieve sustained abstinence. Subsequently, patients who are highly susceptible to relapse should be placed on a maintenance regimen of Suboxone for one or more years in order to achieve sustained abstinence. The characteristics of patients that are highly susceptible to relapse include: a history of substance abuse, mental illness, or childhood sexual abuse or neglect, experiencing chronic pain, and producing low baseline withdrawal scores. Those who are less susceptible to relapse, such as patients who do not have a history of substance abuse but produce high baseline withdrawal scores, may still benefit from maintenance regimens for at least one year.

Once opioid-dependent patients enter into treatment such as behavioral and medicinal therapy (e.g. Suboxone) their chances of successfully recovering from drug abuse increases. Receiving treatment from trained Suboxone detoxification professionals is critical as incorrectly taking Suboxone or discontinuing the medication abruptly can cause severe withdrawal symptoms that will render the treatment ineffective. Patients who are administered a longterm maintenance regimen and consistently attend counseling sessions 
during treatment or seek professional assistance at the first sign of relapse are more likely to fully recover from opioid dependence [28].

With the growth of Suboxone treatments over the past 12 years, this has become a proven therapy for opioid addiction and abuse. The combination of two drugs with prior uses, buprenorphine and naloxone, has resulted in a novel therapy that is both efficacious and carries reduced risks. In light of these advancements, it is clear that Suboxone will continue to be a popular treatment option for opioid dependency.

\section{References}

1. Fishbain DA, Cole B, Lewis J (2008) What percentage of chronic nonmalignant pain patients exposed to chronic opioid analgesic therapy develop abuse/addiction and/or aberrant drug-related behaviors? A structured evidence-based review. Pain Med 9: 444-459.

2. Turk DC, Swanson KS, Gatchel RJ (2008) Predicting opioid misuse by chronic pain patients: a systematic review and literature synthesis. Clin J Pain 24: 497-508.

3. Boscarino JA, Rukstalis M, Hoffman SN. (2010) Risk factors for drug dependence among out-patients on opioid therapy in a large US healthcare system. Addiction 105: 1776-1782.

4. Ives TJ, Chelminski PR, Hammett-Stabler CA (2006) Predictors of opioid misuse in patients with chronic pain: a prospective cohort study. BMC Health Serv Res 6: 46-56.

5. Liebschutz JM, Saitz R, Weiss RD, et al. (2010) Clinical factors associated with prescription drug use disorder in urban primary care patients with chronic pain. The Journal of Pain 8: 1047-1055.

6. Wasan AD, Butler SF, Budman SH (2007) Psychiatric history and psychologic adjustment as risk factors for aberrant drug-related behavior among patients with chronic pain. Clin J Pain 8: 307-315.

7. Davis WR, Johnson BD (2008) Prescription opioid use, misuse, and diversion among street drug users in New York City. Drug Alcohol Depend 92: 267-276.

8. Abuse, National Institute on Drug Abuse (2012) Principles of drug addiction treatment: A research-based guide. Bethesda, MD: National Institutes of Health

9. Camí J, Farre M (2003) Mechanisms of disease: Drug addiction. N Engl J Med 349: 975-986.

10. Kosten TR, George TP (2002) The neurobiology of opioid dependence: implications for treatment. Sci Pract Perspect 1: 13-20.

11. Ling W, Jacobs P, Hillhouse M (2010) From research to the real world: Buprenorphine in the decade of the Clinical Trials Network. J Substance Abuse Treat 38: S53-S60.

12. Polomeni P, Schwan R (2014) Management of opioid addiction with buprenorphine: French history and current management. Int J Gen Med 7: 143-148.

13. Walsh SL, Eissenberg $T$ (2003) The clinical pharmacology of buprenorphine: extrapolating from the laboratory to the clinic. Drug Alcohol Depend 70: S13-S27.

14. Pirnay S, Borron SW, Giudicelli CP (2004) A critical review of the causes of death among post-mortem toxicological investigations: Analysis of 34 buprenorphine-associated and 35 methadone-associated deaths. Addiction 99: 978-988.
15. Polsky D, Glick HA, Woody GE (2010) Cost-effectiveness of Extended Buprenorphine-Naloxone Treatment for Opioid-Dependent Youth: Data from a Randomized Trial. Addiction 105: 1616-1624.

16. Sittambalam CD, Vij R, Ferguson RP (2014) Buprenorphine Outpatient Outcomes Project: can Suboxone be a viable outpatient option for heroin addiction? J Community Hosp Intern Med Perspect 4: 22902.

17. Yokell MA, Zaller ND, Rich JD (2012) Intravenous use of illicit buprenorphine/naloxone to reverse an acute heroin overdose. J Opioid Manag 8: 63-66.

18. Cascade EF, Randall P, Stine S. (2007) Trend watch: Prescribing for buprenorphine in the treatment of opioid addiction. Psychiatry 4: 15-16.

19. Fudala PJ, Bridge TP, Herbert S (2003) Office-based treatment of opiate addiction with a sublingual-tablet formulation of buprenorphine and naloxon. N Engl J Med 349 949-958.

20. Digiusto E, Shakeshaft A, Ritter A, NEPOD Research Group (2004) Serious adverseevents in the Australian National Evaluation of Pahrmacotherapies for Opioid Dependence (NEPOD). Addiction 99: 450-460.

21. Bell JR, Butler B, Lawrance A (2009) Comparing overdose mortality associated with methadone and buprenorphine treatment. Drug Alcohol Depend 104: 73-77.

22. Blum K, Chen TJ, Bailey J (2011) Can the chronic administration of the combination of buprenorphine and naloxone block dopaminergic activity causing anti-reward and relapse potential? Mol Neurobiol 44: 250-268.

23. Ziedonis DM, Amass L, Steinberg M (2009) Predictors of outcome for short-term medically supervised opioid withdrawal during a randomized, multicenter trial of buprenorphine-naloxone and clonidine in the NIDA clinical trials network drug and alcohol dependence. Drug Alcohol Depend 99: 28-36.

24. Ling W, Amass L, Shoptow M, et al. (2005) A multi-center randomized trial of buprenorphine-naloxone versus clonidine for opioid detoxification: Findings from the National Institute on Drug Abuse's Clinical Trial Network. Addiction 100: 1090-1100.

25. Amass L, Ling W, Freese TE (2005) Bringing buprenorphine-naloxone detoxification to community treatment programs: The NIDA Clinical Trial Network field experience. Am J Addict 13: S42-S66.

26. Brigham GS, Amass L, Winhusen T (2007) Using buprenorphine shortterm taper to facilitate early treatment engagement. J Subst Abuse Treat 32: 349-356.

27. Collins ED, Horton T, Reinke K (2007) Using buprenorphine to facilitate entry into residential therapeutic community rehabilitation. J Subst Abuse Treat 32: 167-175.

28. Katz EC, Schwartz RP, King S (2009) Brief vs. extended buprenorphine detoxification in a community treatment program: engagement and short-term outcomes. Am J Drug Alcohol Abuse 35: 63-67.

29. Ling W, Hillhouse M, Domier C (2009) Buprenorphine tapering schedule and illicit opioid use. Addiction 104: 256-265.

30. Woody GE, Poole SA, Subramaniam G (2008) Extended vs. short-term buprenorphine-naloxone for treatment of opioid-addicted youth: A randomized trial. JAMA 300: 2003-2011.

31. Bilban M, Kastelic A, Zaletel-Kragelj L (2008) Ability to work and employability of patients in opioid substitution treatment programs in Slovenia. Croat Med J 49: 842-852. 